\title{
POISSON RANDOM BALLS: SELF-SIMILARITY AND X-RAY IMAGES
}

\author{
HERMINE BIERMÉ $* * *$ AND \\ ANNE ESTRADE, **** Université René Descartes
}

\begin{abstract}
We study a random field obtained by counting the number of balls containing a given point when overlapping balls are thrown at random according to a Poisson random measure. We describe a microscopic process which exhibits multifractional behavior. We are particularly interested in the local asymptotic self-similarity (LASS) properties of the field, as well as in its X-ray transform. We obtain two different LASS properties when considering the asymptotics either in law or in the sense of second-order moments, and prove a relationship between the LASS behavior of the field and the LASS behavior of its $\mathrm{X}$-ray transform. These results can be used to model and analyze porous media, images, or connection networks.
\end{abstract}

Keywords: Random field; random set; shot noise; overlapping spheres; Poisson point process; X-ray transform; asymptotic self-similarity; fractional Brownian motion

2000 Mathematics Subject Classification: Primary 60G60; 60D05; 52A22; 44A12

Secondary 60G12; 60G55; 60G57; 60G18; $60 \mathrm{H} 05$

\section{Introduction}

The purpose of this paper is the study of a random field obtained by throwing overlapping balls. Such a field is particularly well adapted for modeling three-dimensional porous or heterogeneous media. In fact, we consider a collection of balls in $\mathbb{R}^{3}$ whose centers and radii are chosen at random according to a Poisson random measure on $\mathbb{R}^{3} \times \mathbb{R}^{+}$. Equivalently, we consider a germ-grain model where the germs are Poisson distributed and the grains are balls of random radius.

The field under study, commonly known as a shot noise, is the mass density defined as the number of balls containing each point: the more balls covering a given point, the higher is the mass density at this point. From a mathematical point of view, the dimension-three case does not yield any specific behavior, so the study will be carried out in dimension $d \geq 1$. Let us quote, for instance, that for $d=2$ the number of balls covering each point defines the discretized gray level of each pixel in a black-and-white picture. A one-dimensional $(d=1)$ germ-grain model is also relevant for modeling communications networks: the germs represent the starting times of the individual ON periods (calls) and the grains represent the 'half-ball' intervals of duration. The process obtained is a counter which, at each time, delivers the number of active connections in the network.

Received 1 November 2005; revision received 11 September 2006.

* Postal address: MAP5-UMR 8145, Université René Descartes, 45, rue des Saints-Pères, F 75270 Paris cedex 06, France.

** Email address: hermine.bierme@math-info.univ-paris5.fr

*** Email address: anne.estrade@univ-paris5.fr 
We have in mind a microscopic model which yields a macroscopic self-similarity property. In order to obtain this scaling property, we introduce some power law behavior in the radius distribution and consider Poisson random measures on $\mathbb{R}^{d} \times \mathbb{R}^{+}$with intensities of the type

$$
v(\mathrm{~d} \xi, \mathrm{d} r)=C r^{-h} \mathrm{~d} r \mathrm{~d} \xi
$$

for some $h$ which may depend on the location $\xi$ and some constant $C>0$. The origin of the random-balls model described in this paper can be found in the 'micropulses' model introduced by Cioczek-Georges and Mandelbrot [7] with a fixed power $h$ in the intensity measure. The idea is not new, but appeared eighty years ago when Wicksell [19] introduced a first model, the famous 'corpuscles' model, made of random three-dimensional balls defined as above. The aim of his study was to answer a stereological question. Since then, this kind of model has been extensively deepened and extended. We refer the reader to [18, Chapters 3 and 6] or [17, Chapter XIII] for many examples of random models based on Poisson point process, germ-grain, or shot noise models. Let us also mention two recent papers dealing with similar questions. A onedimensional germ-grain model with locations (arrival times) uniformly distributed on the time axis and interval lengths (call durations) given by a power law was considered by Cohen and Taqqu in [8]. A mixed moving average was performed that sums the height of connections, and the so-called Poissonized telecom process was obtained. Also similar is the model recently studied by Kaj et al. [12]: there the germs were uniformly chosen at random in $\mathbb{R}^{d}$ and the grains obtained by random dilation of a fixed, bounded set. In contrast with the quoted models, let us point out that our model is not stationary since we choose a nonstationary intensity measure (1.1) with a nonconstant power $h \equiv h(\xi)$.

This paper is not only concerned with the presentation of a model for random media; we also propose two methods of analyzing the random media mass intensity. On the one hand, self-similarity properties are explored; more precisely, we focus on a parameter that is supposed to contain tangible information on the structure of the media, the local asymptotic self-similar index, or LASS index. On the other hand, the action of an X-ray transform on the field is investigated. This transform is the mathematical interpretation of a radiographic process. These techniques are inspired by those created for Gaussian fields and are still valid in the Poisson context. More specifically, we turn to [6], where anisotropic Gaussian fields were analyzed by performing X-ray transforms and evaluating LASS indices. The fundamental aim of these methods is to make a three-dimensional parameter directly tractable from X-ray images of the media.

The notion of local asymptotic self-similarity was introduced in [3] in a Gaussian context and extended to the non-Gaussian realm in [13] and [4], where a general presentation was given for fields with stationary increments. The LASS index can also be related to other parameters of interest, such as roughness index [2] or Hausdorff dimension [1]. In the area of network modeling the notion of self-similarity, at small or large scales, is also fundamental, and closely connected to long-range dependence. The usual self-similarity property requires a scale invariance in distribution, valid for all scales. This is quite restrictive and we will deal with self-similarity properties that are fulfilled 'at small scales' only.

We now introduce a slight refinement of the LASS property of [3].

Definition 1.1. Let $X=\left\{X(x): x \in \mathbb{R}^{d}\right\}$ be a random field and let $x_{0} \in \mathbb{R}^{d}$. We call the distribution LASS (FDD-LASS) index of $X$ at the point $x_{0}$, denoted by $H_{\mathrm{FDD}}$, the supremum of $\alpha \geq 0$ such that

$$
\lambda^{-\alpha}\left(\Delta_{x_{0}} X(\lambda \cdot)-\mathrm{E}\left(\Delta_{x_{0}} X(\lambda \cdot)\right)\right) \stackrel{\text { FDD }}{\longrightarrow} 0 \quad \text { as } \lambda \downarrow 0,
$$


where $\Delta_{x_{0}} X$ denotes the field of increments at $x_{0}$,

$$
\Delta_{x_{0}} X(x)=X\left(x_{0}+x\right)-X\left(x_{0}\right)
$$

and ' $\stackrel{\text { FDD }}{\longrightarrow}$ ' denotes convergence in finite-dimensional distributions.

Let us remark that convergence in distribution towards the constant 0 is equivalent to convergence in probability. More precisely, $H_{\mathrm{FDD}}$ is also equal to the supremum of $\alpha \geq 0$ such that

$$
\lambda^{-\alpha}\left(\Delta_{x_{0}} X(\lambda x)-\mathrm{E}\left(\Delta_{x_{0}} X(\lambda x)\right)\right) \stackrel{\mathrm{P}}{\rightarrow} 0 \quad \text { as } \lambda \downarrow 0, \quad x \in \mathbb{R}^{d} .
$$

When $H=H_{\mathrm{FDD}}\left(X, x_{0}\right)$ is finite and the finite-dimensional distributions of the centered and renormalized increments $\lambda^{-H}\left(\Delta_{x_{0}} X(\lambda \cdot)-\mathrm{E}\left(\Delta_{x_{0}} X(\lambda \cdot)\right)\right)$ converge to the finite-dimensional distributions of a nonvanishing field as $\lambda \downarrow 0$, the limit field is called the tangent field at point $x_{0}$ (see [9]).

When dealing with real-world data, it is almost impossible to see whether such a limit exists in distribution. We therefore introduce another asymptotic self-similarity property, which only uses the second-order moment.

Definition 1.2. Let $X=\left\{X(x): x \in \mathbb{R}^{d}\right\}$ be a random field and let $x_{0} \in \mathbb{R}^{d}$. We call the covariance LASS (COV-LASS index) index of $X$ at the point $x_{0}$, denoted by $H_{\mathrm{Cov}}\left(X, x_{0}\right)$, the supremum of $\alpha \geq 0$ such that

$$
\lambda^{-2 \alpha} \operatorname{cov}\left(\Delta_{x_{0}} X(\lambda x), \Delta_{x_{0}} X\left(\lambda x^{\prime}\right)\right) \rightarrow 0 \quad \text { as } \lambda \downarrow 0, \quad x, x^{\prime} \in \mathbb{R}^{d} .
$$

By analogy with the situation for $H_{\mathrm{FDD}}$, when $H=H_{\mathrm{COV}}$ is finite and the covariance function of $\lambda^{-H} \Delta_{x_{0}} X(\lambda \cdot)$ converges to a nonvanishing covariance function as $\lambda \downarrow 0$, the limit covariance will be called the tangent covariance at point $x_{0}$.

Note that the above self-similarity indices are equal for Gaussian fields but not in a general setting. Note also that the existence of a tangent covariance does not imply the existence of the tangent field, and vice versa. Actually, if $H_{\mathrm{COV}}$ is the COV-LASS index for $X$ at point $x_{0}$, then the covariance function of $\lambda^{-H} \Delta_{x_{0}} X(\lambda \cdot)$ converges to 0 as $\lambda \downarrow 0$, for all $H<H_{\mathrm{Cov}}$. Thus, the finite-dimensional distributions of its centered version also converge to 0 as $\lambda \downarrow 0$, and the FDD-LASS index for $X$ at point $x_{0}$ - if it exists - satisfies $H_{\mathrm{FDD}} \geq H_{\mathrm{COV}}$.

Our main results can be summarized as follows.

- The proposed models provide microscopic descriptions of macroscopic, asymptotically self-similar fields which look like (multi)fractional Brownian motions, depending on the involved intensity measure.

- In contrast to the Gaussian case, the covariance LASS index and distribution LASS index are not equal: the first can be finite while the second is infinite, or they can take different, finite values.

- The asymptotic distributions are not necessarily Gaussian.

- We obtain explicit formulae that link the LASS indices of a field and the LASS indices of its X-ray transform. In particular, when inhomogeneity or anisotropy is introduced into the model, its presence can be inferred from the LASS indices.

The paper is organized as follows. The random-balls model, i.e. the field that counts the number of balls covering each point, is introduced in Section 2. The intensities of the 
Poisson random measures we will use are prescribed by (1.1) for small radii. A constant power $h \equiv M$ will yield a field which is asymptotically stationary, isotropic, and (mono)fractional. A nonconstant power $h(\xi)$ will yield a multifractional model. We also introduce, in Section 2.2, the X-ray transform. Section 3 is devoted to the scaling properties of the random-balls model and its X-ray transform. Theorems 3.1 and 3.2 deal with the LASS properties in the respective cases where $h(\xi)$ is a smooth function and a singular function. We also compare our results to homogenization results, in Section 3.2. In Section 4 we present some extensions of our model. The proofs of Theorems 3.1 and 3.2 are detailed in Appendix A.

\section{The random-balls model and its $X$-ray transform}

\subsection{The random-balls model}

As in [12], we want to study the mass distribution generated by a family of balls $B\left(\xi_{j}, r_{j}\right)$ with random centers $\xi_{j}$ and random radii $r_{j}$. We assume that the $\left(\xi_{j}, r_{j}\right)$ are given by a Poisson point process with intensity $\nu(\mathrm{d} \xi, \mathrm{d} r)$, where $\nu$ is a nonnegative, $\sigma$-finite measure on $\mathbb{R}^{d} \times \mathbb{R}^{+}$. Inspired by [7], we assume that the radii of such a random grain model obey a power law. Following a widespread idea [15], [3], [6], we assume that the exponent of the power law can depend on the location, $\xi$, of the center of the ball. We define the field $X$ that provides, at each point $x \in \mathbb{R}^{d}$, the number of balls $B(\xi, r)$ that contain the point $x$, namely

$$
X(x)=\operatorname{card}\left\{j: x \in B\left(\xi_{j}, r_{j}\right)\right\}=\sum_{j} \mathbf{1}_{B\left(\xi_{j}, r_{j}\right)}(x)=\int_{\mathbb{R}^{d} \times \mathbb{R}^{+}} \mathbf{1}_{B(\xi, r)}(x) N(\mathrm{~d} \xi, \mathrm{d} r),
$$

where $N$ is a Poisson measure with intensity $v$ such that

$$
\int_{\mathbb{R}^{d} \times \mathbb{R}^{+}} \mathbf{1}_{B(\xi, r)}(x) v(\mathrm{~d} \xi, \mathrm{d} r)<\infty .
$$

We consider intensity measures $v$ satisfying the following assumptions.

- $v(\mathrm{~d} \xi, \mathrm{d} r)=F(\xi, r) \mathrm{d} \xi \mathrm{d} r$ for some nonnegative, measurable function $F$ on $\mathbb{R}^{d} \times \mathbb{R}^{+}$.

- There exists a real function $h$, defined almost everywhere (a.e.) on $\mathbb{R}^{d}$, such that for all $\varepsilon>0$ there exists a $\delta>0$ such that, for a.e. $(\xi, r) \in \mathbb{R}^{d} \times \mathbb{R}^{+}$,

$$
\left|F(\xi, r)-r^{-h(\xi)}\right| \leq \varepsilon r^{-h(\xi)}
$$

for all $r \leq \delta$.

- There exists an $M<d+1$ such that

$$
h(\xi) \leq M \quad \text { a.e. } \quad \text { and } \quad F(\xi, r) \leq C r^{-M} \text { a.e. }
$$

We call the field $X=\left\{X(x): x \in \mathbb{R}^{d}\right\}$ the random-balls model with index $h$. Note that $X$ admits moments of all order. In particular, its mean value and its covariance function are respectively given by

$$
\begin{aligned}
\mathrm{E}(X(x)) & =\int_{\mathbb{R}^{d} \times \mathbb{R}^{+}} \mathbf{1}_{B(\xi, r)}(x) v(\mathrm{~d} \xi, \mathrm{d} r), \\
\operatorname{cov}\left(X(x), X\left(x^{\prime}\right)\right) & =\int_{\mathbb{R}^{d} \times \mathbb{R}^{+}} \mathbf{1}_{B(\xi, r)}(x) \mathbf{1}_{B(\xi, r)}\left(x^{\prime}\right) \nu(\mathrm{d} \xi, \mathrm{d} r) .
\end{aligned}
$$




\subsection{X-ray transform}

One motivation for this paper is to describe, model, and analyze heterogeneous media. We have in mind the possibility of studying their three-dimensional behavior using X-ray images, by which means it will be possible to perform an analysis of the media without entering it (a noninvasive method). In this section the mathematical tool associated with X-ray images is presented and tested on the random-balls model. We assume that $d \geq 2$.

Following the usual notation (see [16, p. 13], for instance), the X-ray transform of a function $f \in L^{1}\left(\mathbb{R}^{d}\right)$ in the direction $\alpha \in S^{d-1}=\left\{x \in \mathbb{R}^{d}:\|x\|=1\right\}$ is given by $\langle\alpha\rangle^{\perp} \ni y \mapsto$ $\int_{\mathbb{R}} f(y+p \alpha) \mathrm{d} p$, where $\langle\alpha\rangle^{\perp}:=\left\{x \in \mathbb{R}^{d}: x \cdot \alpha=0\right\}$ and '.' denotes the usual scalar product on $\mathbb{R}^{d}$. We are interested in defining a kind of X-ray transform for the random-balls model. We will work with the windowed $\mathrm{X}$-ray transform defined in terms of a fixed window $\rho$. We assume that $\rho$ is a continuous function on $\mathbb{R}$ with fast decay, i.e. for all $N \in \mathbb{N},|\rho(p)| \leq C_{N}(1+|p|)^{-N}$ for all $p \in \mathbb{R}$ and some constant $C_{N}$. For any function $f \in L^{1}\left(\mathbb{R}^{d}\right)$ with compact support, we define the windowed $X$-ray transform of $f$ in the direction $\alpha$ to be the map

$$
\langle\alpha\rangle^{\perp} \ni y \mapsto \mathcal{P}_{\alpha} f(y):=\int_{\mathbb{R}} f(y+p \alpha) \rho(p) \mathrm{d} p .
$$

It is straightforward to see that $(\xi, r) \mapsto \mathcal{P}_{\alpha} \mathbf{1}_{B(\xi, r)}(y)$ is integrable with respect to $v(\mathrm{~d} \xi, \mathrm{d} r)$ for each $y \in\langle\alpha\rangle^{\perp}$. Thus, we can define the windowed $X$-ray transform of $X$ in the direction $\alpha$ to be the field given by

$$
\mathcal{P}_{\alpha} X(y):=\int_{\mathbb{R}^{d} \times \mathbb{R}^{+}} \mathcal{P}_{\alpha} \mathbf{1}_{B(\xi, r)}(y) N(\mathrm{~d} \xi, \mathrm{d} r), \quad y \in\langle\alpha\rangle^{\perp} .
$$

Note that, for $y \in\langle\alpha\rangle^{\perp}$, by the Cauchy-Schwarz inequality, $\mathcal{P}_{\alpha} \mathbf{1}_{B(\xi, r)}$ (y) belongs to $L^{2}\left(\mathbb{R}^{d} \times \mathbb{R}^{+}, v(\mathrm{~d} \xi, \mathrm{d} r)\right)$, so $\mathcal{P}_{\alpha} X$ admits a second-order moment.

\section{Scaling properties}

\subsection{Self-similarity properties}

In this section we study the LASS properties of a random-balls model with index function $h$ and, simultaneously, the LASS properties of its X-ray transform. We are looking for links between the LASS indices and the index $h$ of the random-balls model.

When dealing with the COV-LASS properties, we have to study, in particular, the asymptotic behavior of $\operatorname{var}\left(\Delta_{x_{0}} X(\lambda x)\right)$ as $\lambda \downarrow 0$, for all $x_{0}, x \in \mathbb{R}^{d}$. By a change of variables,

$$
\operatorname{var}\left(\Delta_{x_{0}} X(\lambda x)\right)=\int_{\mathbb{R}^{d} \times \mathbb{R}^{+}} \lambda^{d+1}\left(\mathbf{1}_{B(\xi, r)}(x)-\mathbf{1}_{B(\xi, r)}(0)\right)^{2} F\left(x_{0}+\lambda \xi, \lambda r\right) \mathrm{d} \xi \mathrm{d} r .
$$

Since we want to replace $F\left(x_{0}+\lambda \xi, \lambda r\right)$ by $(\lambda r)^{-h\left(x_{0}+\lambda \xi\right)}$, it appears that further assumptions on $h$ have to be made. We are mainly interested in two kinds of index functions. The first kind are smooth on $\mathbb{R}^{d}$, and are linked with the multifractional Brownian motion [15], [3] obtained by substituting the Hurst parameter $H$ by a Hölder regular function on the state space. The second class is of functions $h(\xi)$ that depend only on the direction of $\xi$, which induces a singularity at the point 0 . We consider a Hölder regular function on the sphere extended onto $\mathbb{R}^{d} \backslash\{0\}$ by taking $h(\xi)=h(\xi /\|\xi\|)$, i.e. $h(\lambda \xi)=h(\xi)$ for all $\lambda \in \mathbb{R} \backslash\{0\}$. This follows the point of view taken in [6] to obtain anisotropic generalizations of the fractional Brownian motion.

Let us recall the definition of a $\beta$-Hölder function. 
Definition 3.1. Let $\left(E, d_{E}\right)$ be a metric space and let $\beta \in(0,1]$. A function $f: E \rightarrow \mathbb{R}$ is called $\beta$-Hölder on $E$ if there exists a $C>0$ such that, for any $x, y \in E$ with $d_{E}(x, y) \leq 1$,

$$
|f(x)-f(y)| \leq C d_{E}(x, y)^{\beta} .
$$

3.1.1. The smooth case. Let us first study the case of a $\beta$-Hölder function on $\mathbb{R}^{d}$. By continuity of $h$ around $x_{0} \in \mathbb{R}^{d}$, it intuitively follows from (3.1) that the COV-LASS index of $X$ at point $x_{0}$ is equal to $\left(d+1-h\left(x_{0}\right)\right) / 2$. Moreover, similar arguments can be applied to $\mathcal{P}_{\alpha} X$, the $\mathrm{X}$-ray transform of $X$ with the window $\rho$, given by (2.3). The COV-LASS index is equal to $\left(d+2-M\left(\alpha, y_{0}\right)\right) / 2$, with

$$
M\left(\alpha, y_{0}\right):=\sup \left\{h\left(y_{0}+t \alpha\right): t \in \operatorname{supp} \rho\right\}
$$

where supp $\rho$ denotes the support of $\rho$. This suggests the following theorem, whose detailed proof is given in Section A.1. We denote by meas(.) the Lebesgue measure.

Theorem 3.1. Let $d \geq 2$. Let h be a $\beta$-Hölderfunction on $\mathbb{R}^{d}$ such that $d<h<d+1$. Let $X$ be a random-balls model with index $h$ and let $\mathcal{P}_{\alpha} X$ be its windowed $X$-ray transform in the direction $\alpha \in S^{d-1}$. The following statements then hold.

- At any point $x_{0} \in \mathbb{R}^{d}$,

$$
H_{\mathrm{COV}}\left(X, x_{0}\right)=\frac{d+1-h\left(x_{0}\right)}{2} \text { and } H_{\mathrm{FDD}}\left(X, x_{0}\right) \geq 2 H_{\mathrm{COV}}\left(X, x_{0}\right) .
$$

Moreover, the covariance of $\lambda^{-H_{\mathrm{Cov}}\left(X, x_{0}\right)} \Delta_{x_{0}} X(\lambda \cdot)$ converges, up to a multiplicative constant, to the covariance of a fractional Brownian motion of index $H_{\mathrm{COV}}\left(X, x_{0}\right)$.

- At any point $y_{0} \in\langle\alpha\rangle^{\perp}$,

$$
H_{\mathrm{COV}}\left(\mathcal{P}_{\alpha} X, y_{0}\right)=H_{\mathrm{FDD}}\left(\mathcal{P}_{\alpha} X, y_{0}\right)=\frac{d+2-M\left(\alpha, y_{0}\right)}{2} .
$$

Moreover, when meas $\left(\left\{t \in \operatorname{supp} \rho: h\left(y_{0}+t \alpha\right)=M\left(\alpha, y_{0}\right)\right\}\right)>0$, the covariance and the finite-dimensional distributions of

$$
\lambda^{-H_{\mathrm{Cov}}\left(\mathcal{P}_{\alpha} X, y_{0}\right)}\left(\Delta_{y_{0}}\left(\mathcal{P}_{\alpha} X\right)(\lambda \cdot)-\mathrm{E}\left(\Delta_{y_{0}}\left(\mathcal{P}_{\alpha} X\right)(\lambda \cdot)\right)\right)
$$

respectively converge, up to a multiplicative constant, to those of a fractional Brownian motion of index $H_{\mathrm{COV}}\left(\mathcal{P}_{\alpha} X, y_{0}\right)$.

Remark 3.1. (Concerning the first point of Theorem 3.1.) First note that the first point of Theorem 3.1 is still true in the one-dimensional case $(d=1)$. This result describes the smallscale behavior of the number of active connections in a communications network: the covariance is locally asymptotically self-similar and behaves like a fractional Brownian motion covariance. More generally, the same is observed in the multidimensional case. Hence, the random-balls model provides a microscopic description of random media which behave, up to the secondorder moment, like multifractional Brownian motion.

Concerning the FDD-LASS property, let us point out that $H_{\mathrm{FDD}} \neq H_{\mathrm{COV}}$. Moreover, it is straightforward to see that $H_{\mathrm{FDD}}=\infty$ when $F(\xi, r)=r^{-M} \mathbf{1}_{(0,1)}(r)$. 
Remark 3.2. (Concerning the second point of Theorem 3.1.) From a practical point of view, the correspondence between the COV-LASS index of $X$ and the COV-LASS index of $\mathcal{P}_{\alpha} X$ allows for the estimation of the three-dimensional LASS index through the analysis of radiographic images. However, note that only the suprema of $h$ along straight lines in the support of $\rho$ can be recovered.

Remark 3.3. (Concerning the case in which $h=M$ is constant.) When $h=M$ is constant, there is a one-to-one correspondence between the COV-LASS indices at any points:

$$
H_{\mathrm{COV}}\left(\mathcal{P}_{\alpha} X, y_{0}\right)=\frac{d+2-M}{2}=H_{\mathrm{COV}}\left(X, x_{0}\right)+\frac{1}{2} .
$$

Note that the same link between the LASS indices was obtained in [6] for Gaussian fields of fractional Brownian type.

3.1.2. The singular case. Now let us assume $h$ to be an even $\beta$-Hölder function on the sphere extended onto $\mathbb{R}^{d} \backslash\{0\}$ by taking $h(\xi)=h(\xi /\|\xi\|)$ and choosing any value for $h(0)$. Let us remark that, unless $h$ is constant, there is no way to extend $h$ continuously at the point 0 . To distinguish the random-balls model associated with such a singular index $h$ from the one associated with a smooth $h$, we will call it the singular random-balls model. Let $x_{0} \in \mathbb{R}^{d} \backslash\{0\}$, let $\xi \in \mathbb{R}^{d}$, and note that

$$
\left\|\frac{x_{0}+\xi}{\left\|x_{0}+\xi\right\|}-\frac{x_{0}}{\left\|x_{0}\right\|}\right\| \leq 2 \frac{\|\xi\|}{\left\|x_{0}\right\|} \quad \text { for }\|\xi\| \leq \frac{\left\|x_{0}\right\|}{2} .
$$

Thus, the $\beta$-Hölder assumption on the sphere and the boundedness of $h$ imply that there exists a $C>0$ such that

$$
\left|h\left(x_{0}+\xi\right)-h\left(x_{0}\right)\right| \leq C\left\|x_{0}\right\|^{-\beta}\|\xi\|^{\beta} .
$$

Thus, given that $x_{0}$ is not 0 , the LASS properties of the singular random-balls model at $x_{0}$ are the same as those of the smooth model, given in Theorem 3.1. The next theorem will therefore only deal with the LASS properties around 0 .

Theorem 3.2. Let $h$ be an even, nonconstant $\beta$-Hölder function on $S^{d-1}$ such that $d<h \leq$ $M=\max _{S^{d-1}} h<d+1$. Let $X$ be the singular random-balls model with index $h$ and let $\mathcal{P}_{\alpha} X$ be its $X$-ray transform in the direction $\alpha \in S^{d-1}$. The following statements then hold.

- For $H=(d+1-M) / 2$,

$$
H_{\mathrm{COV}}(X, 0)=H \quad \text { and } \quad H_{\mathrm{FDD}}(X, 0)=2 H .
$$

When meas $(\{h=M\})>0$, the covariance of $\lambda^{-H} \Delta_{0} X(\lambda \cdot)$ converges to

$$
\begin{aligned}
& \Gamma_{H}\left(x, x^{\prime}\right)=\int_{\mathbb{R}^{d} \times \mathbb{R}^{+}} \mathbf{1}_{\{h(\xi)=d+1-2 H\}} \psi(x, \xi, r) \psi\left(x^{\prime}, \xi, r\right) r^{-d-1+2 H} \mathrm{~d} \xi \mathrm{d} r, \\
& x, x^{\prime} \in \mathbb{R}^{d},
\end{aligned}
$$

while the finite-dimensional distributions of $\lambda^{-2 H}\left(\Delta_{0} X(\lambda \cdot)-\mathrm{E}\left(\Delta_{0} X(\lambda \cdot)\right)\right)$ converge to the deterministic field

$$
Z_{H}(x)=-\|x\|^{2 H} \int_{\mathbb{R}^{d} \times \mathbb{R}^{+}} \mathbf{1}_{\{h(\xi)=d+1-2 H\}} \psi\left(\frac{x}{\|x\|}, \xi, r\right) r^{-d-1+2 H} \mathrm{~d} \xi \mathrm{d} r,
$$

$$
x \in \mathbb{R}^{d} .
$$


Here $\psi$ is given by

$$
\psi(x, \xi, r)=\mathbf{1}_{B(\xi, r)}(x)-\mathbf{1}_{B(\xi, r)}(0)=\mathbf{1}_{\{\|x-\xi\|<r \leq\|\xi\|\}}-\mathbf{1}_{\{\|\xi\|<r \leq\|x-\xi\|\}} .
$$

- $H_{\mathrm{COV}}\left(\mathcal{P}_{\alpha} X, 0\right)=H_{\mathrm{FDD}}\left(\mathcal{P}_{\alpha} X, 0\right)=(d+2-h(\alpha) / 2)$. Moreover, the covariance and the finite-dimensional distributions of $\lambda^{-H_{\mathrm{Cov}}\left(\mathcal{P}_{\alpha} X, 0\right)}\left(\Delta_{0}\left(\mathcal{P}_{\alpha} X\right)(\lambda \cdot)-\mathrm{E}\left(\Delta_{0}\left(\mathcal{P}_{\alpha} X\right)(\lambda \cdot)\right)\right)$ respectively converge, up to a multiplicative constant, to those of a fractional Brownian motion of index $H_{\mathrm{COV}}\left(\mathcal{P}_{\alpha} X, 0\right)$.

Let us remark that, for the singular random-balls model, there exist both a COV-LASS index and a FDD-LASS index, and that the latter equals twice the former. This multiplicative factor is typical given the Poisson structure, since for Poisson random variables the variance equals the mean.

Moreover, when $\{h=M\}$ has positive measure, the tangent field at 0 is deterministic and nonzero, and hence does not have stationary increments. This result is linked to a result of Falconer [9] which states that at almost all points the tangent field - if it exists - must have stationary increments. The point 0 therefore appears as an 'exceptional point' (see [14] for other examples of exceptional points).

Finally, let us point out that the tangent field of the X-ray transform, when it exists, is Gaussian - perhaps even fractional Brownian motion - whereas the tangent field of the singular random-balls model is deterministic. This can justify, from a mathematical point of view, the modeling of radiographic images using fractional Brownian motion even when the media under study are far from being of this type (see [10] for an experimental study).

\subsection{Comparison with homogenization results}

There are different ways to consider self-similarity at small scales, depending on which part of the signal is concerned with the scaling. Instead of performing a scaling on the increments lag, as done in Section 3.1, we act on the radius of the balls as follows. Suppose we zoom in and consider the balls $B(\xi, r / \varepsilon)$ instead of the balls $B(\xi, r)$, where the $(\xi, r)$ are randomly chosen by the Poisson random measure $N$ and we let $\varepsilon$ decrease to 0 . Denoting by $X^{\varepsilon}$ the associated field

$$
X^{\varepsilon}(x)=\int_{\mathbb{R}^{d} \times \mathbb{R}^{+}} \mathbf{1}_{B(\xi, r / \varepsilon)}(x) N(\mathrm{~d} \xi, \mathrm{d} r), \quad x \in \mathbb{R}^{d},
$$

we look for normalization terms $n\left(x_{0}\right)$ such that $\varepsilon^{n\left(x_{0}\right)}\left(\Delta_{x_{0}} X^{\varepsilon}-\mathrm{E}\left(\Delta_{x_{0}} X^{\varepsilon}\right)\right)$ converges in distribution to a nondegenerate field. Note that the field $X^{\varepsilon}$ can also be considered to be a random-balls model associated with a Poisson measure with intensity

$$
\nu^{\varepsilon}(\mathrm{d} \xi, \mathrm{d} r)=\varepsilon F(\xi, \varepsilon r) \mathrm{d} \xi \mathrm{d} r .
$$

Actually, this procedure is nothing but homogenization and is close to the thermodynamical limit investigated in [7] and the scaling limit in [11] or [12]. Computations similar to those for the previous theorems yield the following results.

- If $d<M:=\max _{S^{d-1}} h<d+1$ and the set $\{\xi: h(\xi)=M\}$ has positive measure, then the normalization term $n\left(x_{0}\right)$ is equal to $(M-1) / 2$ for all $x_{0} \in \mathbb{R}^{d}$.

- Moreover, if $h=M$ is constant, then the limit field is a fractional Brownian motion with index $(d+1-M) / 2$ (see [5] for similar ideas). 


\section{Conclusion and more general setting}

We have proposed to model, from a microscopic point of view, the mass intensity of porous media or the number of connected customers in a network using a non-Gaussian field that exhibits macroscopic (multi)fractional behavior. The rich structure of Poisson point processes allows us to reach this goal and also to perform explicit computations, as in the Gaussian case. In order to keep the model as intuitively clear as possible, we have not introduced more general fields. The Poisson structure can obviously be exploited further by considering more general integrators with respect to the Poisson measure. Replacing the ball $B(\xi, r)$ by a more general set will, for instance, allow one to model granular media with nonspherical grains. One can also consider a general function $f(\cdot-\xi, r)$ instead of the indicator function $\mathbf{1}_{B(\xi, r)}(\cdot)$, which leads to a nonstationary shot noise process. In order to obtain self-similarity properties under the power law assumption on the intensity measure, one has to consider an integrator $f(\cdot-\xi, r)$ that is asymptotically homogeneous. Another model for porous media could be built up from a collection of random balls which no longer correspond to grains but to pores or bubbles. This way, one will obtain a $\{0,1\}$-valued field.

\section{Appendix A. Proofs of the LASS properties}

In this section we will give rigorous proofs for the LASS properties of the random-balls models and their windowed X-ray transforms. We will first state a preliminary lemma which allows us to replace the intensity measure by its equivalent when the radius decreases to 0 . Note that assumption (2.1) holds uniformly in $\xi \in \mathbb{R}^{d}$. However, with smoothness assumptions on the function $h$ on $\mathbb{R}^{d}$, we want to replace $F\left(x_{0}+\lambda \xi, \lambda r\right)$ not by $(\lambda r)^{-h\left(x_{0}+\lambda \xi\right)}$ but by $(\lambda r)^{-h\left(x_{0}\right)}$. In fact,

$$
\left|r^{-h\left(x_{0}+\xi\right)}-r^{-h\left(x_{0}\right)}\right| \leq C \mathrm{e}\|\xi\|^{\beta}|\ln (r)| r^{-h\left(x_{0}\right)}
$$

holds for small $r$ and $\xi \in \mathbb{R}^{d}$ such that $\|\xi\|^{\beta}|\ln (r)| \leq 1$, using the $\beta$-Hölder assumption on $h$. We state the next lemma in this general setting.

Lemma A.1. Let $d \geq 1$ and $\beta \in(0,1]$. Let $F, F_{0}$, and $F_{\infty}$ be nonnegative functions on $\mathbb{R}^{d} \times \mathbb{R}^{+}$. Let us assume that, for all $\varepsilon>0$, there exists a $\delta>0$ such that

$$
\left|F(\xi, r)-F_{0}(\xi, r)\right| \leq \varepsilon F_{0}(\xi, r)
$$

for almost every $(\xi, r)$ in $\mathbb{R}^{d} \times \mathbb{R}^{+}$such that $r \leq \delta$ and $\|\xi\|^{\beta}|\ln (r)| \leq \delta$. Let us also assume that $F$ and $F_{0}$ are bounded from above by $F_{\infty}$, which satisfies

$$
F_{\infty}(\xi, r) \leq C r^{-M} \text { a.e. }
$$

for some $M \in(d, d+1)$.

Let $\left(f_{\lambda}\right)$ be a family of functions on $\mathbb{R}^{d} \times \mathbb{R}^{+}$bounded from above by a nonnegative function $f_{\infty}$ such that, for some $q_{0}$ and $q_{1}, q_{0}<M<q_{1}$,

$$
\int_{\mathbb{R}^{d}} f_{\infty}(\xi, r) \mathrm{d} \xi \leq C \min \left(r^{q_{1}-1}, r^{q_{0}-1}\right) \text { a.e. }
$$

and, for all sufficiently large $A>0$,

$$
\int_{\|\xi\|>A} \int_{\mathbb{R}^{+}} f_{\infty}(\xi, r) r^{-M} \mathrm{~d} r \leq C A^{-(M-d)} .
$$


Then, for any $q$ such that $\max \left(q_{0}, d\right)<q<M$ and any $\varepsilon>0$, there exists a $\delta_{0}>0$ such that

$$
\begin{array}{r}
\left|\int_{\mathbb{R}^{d} \times \mathbb{R}^{+}} f_{\lambda}(\xi, r) F(\lambda \xi, \lambda r) \mathrm{d} \xi \mathrm{d} r-\int_{\mathbb{R}^{d} \times \mathbb{R}^{+}} f_{\lambda}(\xi, r) F_{0}(\lambda \xi, \lambda r) \mathrm{d} \xi \mathrm{d} r\right| \\
\quad \leq \varepsilon \int_{\mathbb{R}^{d} \times \mathbb{R}^{+}}\left|f_{\lambda}(\xi, r)\right| F_{0}(\lambda \xi, \lambda r) \mathrm{d} \xi \mathrm{d} r+C \lambda^{-q}
\end{array}
$$

for $\lambda \leq \delta_{0}$.

Proof. Let us choose an $s \in(0,1)$ and an $n \in \mathbb{N}$ with $n \neq 0$, and let us remark that, for $\|\xi\| \leq \lambda^{-s}$ and $r \in\left(\lambda^{n}, \delta \lambda^{-1}\right)$,

$$
\|\lambda \xi\|^{\beta}|\ln (\lambda r)| \leq n \lambda^{\beta(1-s)}|\ln (\lambda)| .
$$

Therefore, for $\varepsilon>0$, we can choose $\lambda$ small enough that

$$
\begin{aligned}
\left|\int_{B\left(0, \lambda^{-s}\right) \times\left(\lambda^{n}, \delta \lambda^{-1}\right)} f_{\lambda}(\xi, r)\left(F(\lambda \xi, \lambda r)-F_{0}(\lambda \xi, \lambda r)\right) \mathrm{d} \xi \mathrm{d} r\right| & \\
& \leq \varepsilon \int_{\mathbb{R}^{d} \times \mathbb{R}^{+}}\left|f_{\lambda}(\xi, r)\right| F_{0}(\lambda \xi, \lambda r) \mathrm{d} \xi \mathrm{d} r .
\end{aligned}
$$

Moreover,

$$
\begin{aligned}
\left|\int_{\|\xi\| \geq \lambda^{-s}} \int_{\left(0, \delta \lambda^{-1}\right)} f_{\lambda}(\xi, r) F(\lambda \xi, \lambda r) \mathrm{d} \xi \mathrm{d} r\right| & \leq C \lambda^{-M} \int_{\|\xi\| \geq \lambda^{-s}} \int_{\mathbb{R}^{+}} f_{\infty}(\xi, r) r^{-M} \mathrm{~d} \xi \mathrm{d} r \\
& \leq C \lambda^{-M-s(d-M)}
\end{aligned}
$$

by (A.1) and (A.3). On the other hand,

$$
\begin{aligned}
\left|\int_{\left(0, \lambda^{n}\right)} \int_{\mathbb{R}^{d}} f_{\lambda}(\xi, r) F(\lambda \xi, \lambda r) \mathrm{d} \xi \mathrm{d} r\right| & \leq C \lambda^{-M} \int_{\left(0, \lambda^{n}\right)} r^{q_{1}-M-1} \mathrm{~d} r \\
& \leq C \lambda^{-M+n\left(q_{1}-M\right)}
\end{aligned}
$$

by (A.1) and (A.2), since $M<q_{1}$. Finally,

$$
\begin{aligned}
\left|\int_{\left(\delta \lambda^{-1}, \infty\right)} \int_{\mathbb{R}^{d}} f_{\lambda}(\xi, r) F(\lambda \xi, \lambda r) \mathrm{d} \xi \mathrm{d} r\right| & \leq C \lambda^{-M} \int_{\left(\delta \lambda^{-1}, 1\right)} r^{q_{0}-M-1} \mathrm{~d} r \\
& \leq C \delta^{q_{0}-M} \lambda^{-q_{0}}
\end{aligned}
$$

by (A.1) and (A.2), since $M>q_{0}$.

Since the same upper bounds are valid for $F_{0}$, it is sufficient to take $s=(M-q) /(M-d)$ and $n>(M-q) /\left(q_{1}-M\right)$ to complete the proof.

The following corollary will play the role of Lebesgue's theorem to ensure convergence of the integrals.

Corollary A.1. We make the assumptions of Lemma A.1. Let us assume, moreover, that there exist a real number $H \geq M$ and two functions, $f_{0}$ and $F_{0}^{0}$, such that, for almost all $(\xi, r) \in$ $\mathbb{R}^{d} \times \mathbb{R}^{+}$,

$$
\lambda^{H} f_{\lambda}(\xi, r) F_{0}(\lambda \xi, \lambda r) \rightarrow f_{0}(\xi, r) F_{0}^{0}(\xi, r) \text { as } \lambda \downarrow 0 .
$$


Then

$$
\lim _{\lambda \downarrow 0} \int_{\mathbb{R}^{d} \times \mathbb{R}^{+}} \lambda^{H} f_{\lambda}(\xi, r) F(\lambda \xi, \lambda r) \mathrm{d} \xi \mathrm{d} r=\int_{\mathbb{R}^{d} \times \mathbb{R}^{+}} f_{0}(\xi, r) F_{0}^{0}(\xi, r) \mathrm{d} \xi \mathrm{d} r .
$$

Proof. Let $\varepsilon>0$. From Lemma A.1, there exists a $\delta_{0}>0$ such that, for $\lambda \leq \delta_{0}$,

$$
\begin{aligned}
\mid \int_{\mathbb{R}^{d} \times \mathbb{R}^{+}} \lambda^{H} f_{\lambda}(\xi, r) F(\lambda \xi, \lambda r) \mathrm{d} \xi \mathrm{d} r & -\int_{\mathbb{R}^{d} \times \mathbb{R}^{+}} \lambda^{H} f_{\lambda}(\xi, r) F_{0}(\lambda \xi, \lambda r) \mathrm{d} \xi \mathrm{d} r \mid \\
& \leq \varepsilon \int_{\mathbb{R}^{d} \times \mathbb{R}^{+}} \lambda^{H}\left|f_{\lambda}(\xi, r)\right| F_{0}(\lambda \xi, \lambda r) \mathrm{d} \xi \mathrm{d} r+C \lambda^{H-q} .
\end{aligned}
$$

Moreover, by (A.1),

$$
\lambda^{H}\left|f_{\lambda}(\xi, r)\right| F_{0}(\lambda \xi, \lambda r) \leq C \lambda^{H-M} f_{\infty}(\xi, r) r^{-M}
$$

with $f_{\infty}(\xi, r) r^{-M} \in L^{1}\left(\mathbb{R}^{d} \times \mathbb{R}^{+}\right)$according to (A.2). Lebesgue's theorem then implies that

$$
\lim _{\lambda \downarrow 0} \int_{\mathbb{R}^{d} \times \mathbb{R}^{+}} \lambda^{H} f_{\lambda}(\xi, r) F_{0}(\lambda \xi, \lambda r) \mathrm{d} \xi \mathrm{d} r=\int_{\mathbb{R}^{d} \times \mathbb{R}^{+}} f_{0}(\xi, r) F_{0}^{0}(\xi, r) \mathrm{d} \xi \mathrm{d} r,
$$

which yields the result.

\section{A.1. Proof of Theorem 3.1}

Let us first consider the COV-LASS property of the random-balls model. Let $x_{0} \in \mathbb{R}^{d}$. For $H \in(0,1)$ and $\lambda>0$, let us write $\Gamma_{\lambda}^{H}\left(x_{0}, \cdot\right)$ for the covariance function of $\lambda^{-H} \Delta_{x_{0}} X(\lambda \cdot)$. By the same change of variables used to obtain (3.1), for $x, x^{\prime} \in \mathbb{R}^{d}, \Gamma_{\lambda}^{H}\left(x_{0}, x, x^{\prime}\right)$ is equal to

$$
\int_{\mathbb{R}^{d} \times \mathbb{R}^{+}} \lambda^{-2 H+d+1} \psi(\lambda x, \lambda \xi, \lambda r) \psi\left(\lambda x^{\prime}, \lambda \xi, \lambda r\right) F\left(x_{0}+\lambda \xi, \lambda r\right) \mathrm{d} \xi \mathrm{d} r
$$

with $\psi$, given by (3.2), satisfying $\psi(\lambda x, \lambda \xi, \lambda r)=\psi(x, \xi, r)$. In order to apply Corollary A.1, let us check that $\psi$ satisfies assumptions (A.2) and (A.3) of Lemma A.1.

Lemma A.2. Let $M \in(d, d+1)$. There exists a constant $C_{M} \in(0, \infty)$ such that, for all $p \in(0, \infty)$ and $x \in \mathbb{R}^{d}$,

$$
\int_{\mathbb{R}^{d} \times \mathbb{R}^{+}}|\psi(x, \xi, r)|^{p} r^{-M} \mathrm{~d} \xi \mathrm{d} r=C_{M}\|x\|^{d+1-M},
$$

with

$$
\int_{\mathbb{R}^{d}}|\psi(x, \xi, r)|^{p} \mathrm{~d} \xi \leq C(x) \min \left(r^{d}, r^{d-1}\right)
$$

and, for $A>2\|x\|$,

$$
\int_{\|\xi\|>A} \int_{\mathbb{R}^{+}}|\psi(x, \xi, r)|^{p} r^{-M} \mathrm{~d} \xi \mathrm{d} r \leq \frac{2^{M-d}}{M-d} C(x) A^{-(M-d)},
$$

where $C(x)$ is a positive constant that depends on $x$. 
Proof. Note that $|\psi(x, \xi, r)|^{p}=|\psi(x, \xi, r)|=\mathbf{1}_{\{\|x-\xi\|<r \leq\|\xi\|\}}+\mathbf{1}_{\{\|\xi\|<r \leq\|x-\xi\|\}\}}$. Hence, for all $x, \xi \in \mathbb{R}^{d}$, the integral $\int_{\mathbb{R}^{+}}|\psi(x, \xi, r)|^{p} r^{-M} \mathrm{~d} r$ is equal to

$$
\frac{1}{M-1}\left(\mathbf{1}_{\{\|\xi\|<\|x-\xi\|\}}-\mathbf{1}_{\{\|x-\xi\|<\|\xi\|\}}\right)\left(\|\xi\|^{-M+1}-\|x-\xi\|^{-M+1}\right)
$$

For $d<M<d+1$, the function $\xi \mapsto\|\xi\|^{-M+1}-\|x-\xi\|^{-M+1}$ is integrable on $\mathbb{R}^{d}$. The equality is obtained by rotational invariance and homogeneity.

Let us prove (A.4) and (A.5). It is straightforward to see that

$$
\int_{\mathbb{R}^{d}}|\psi(x, \xi, r)|^{p} \mathrm{~d} \xi \leq C\left((r+\|x\|)^{d}-r^{d}\right),
$$

whence (A.4) holds with $C(x)=C \max \left(2^{d}, 2^{d-1} d\right) \max (\|x\|, 1)$. Finally, let us choose $A>2\|x\|$ and note that

$$
\int_{\|\xi\|>A} \int_{\mathbb{R}^{+}}|\psi(x, \xi, r)|^{p} r^{-M} \mathrm{~d} \xi \mathrm{d} r \leq \int_{A / 2}^{\infty} \int_{\mathbb{R}^{d}}|\psi(x, \xi, r)|^{p} r^{-M} \mathrm{~d} \xi \mathrm{d} r
$$

thus, (A.5) holds as a consequence of (A.4), and Lemma A.2 is proved.

Let us remark that, according to (2.1), (2.2), and the $\beta$-Hölder assumption, $F\left(x_{0}+\cdot, \cdot\right)$ satisfies the assumptions of Lemma A.1 with $F_{0}(\xi, r)=r^{-h\left(x_{0}\right)}$. We first establish that, for $H=\left(d+1-h\left(x_{0}\right)\right) / 2$,

$$
\Gamma_{\lambda}^{H}\left(x_{0}, x, x^{\prime}\right) \rightarrow \Gamma^{H}\left(x, x^{\prime}\right) \quad \text { as } \lambda \downarrow 0,
$$

where

$$
\Gamma^{H}\left(x, x^{\prime}\right)=\int_{\mathbb{R}^{d} \times \mathbb{R}^{+}} \psi(x, \xi, r) \psi\left(x^{\prime}, \xi, r\right) r^{-d-1+2 H} \mathrm{~d} \xi \mathrm{d} r .
$$

Then we prove that (up to a constant) $\Gamma^{H}$ is the covariance function of a fractional Brownian motion with Hurst index $H$.

We set $f_{\lambda}(\xi, r)=\psi(\lambda x, \lambda \xi, \lambda r) \psi\left(\lambda x^{\prime}, \lambda \xi, \lambda r\right)=\psi(x, \xi, r) \psi\left(x^{\prime}, \xi, r\right)$. Then $f_{\infty}=\left|f_{\lambda}\right|$ satisfies (A.2) and (A.3) with $q_{0}=d$ and $q_{1}=d-1$, using the Cauchy-Schwartz inequality, (A.4), and (A.5). Moreover, for $f_{0}=f_{\lambda}$,

$$
\lambda^{h\left(x_{0}\right)} f_{\lambda}(\xi, r) F_{0}(\lambda \xi, \lambda r) \rightarrow f_{0}(\xi, r) r^{-h\left(x_{0}\right)} \text { as } \lambda \downarrow 0 .
$$

Thus, (A.6) is obtained from Corollary A.1, for $-2 H+d+1=h\left(x_{0}\right)$. It remains to show that (up to a constant) $\Gamma^{H}$ is the covariance function of a fractional Brownian motion with Hurst index $H=\left(d+1-h\left(x_{0}\right)\right) / 2$. A straightforward computation gives

$$
\int_{\mathbb{R}^{d} \times \mathbb{R}^{+}}\left(\psi(x, \xi, r)-\psi\left(x^{\prime}, \xi, r\right)\right)^{2} r^{-h\left(x_{0}\right)} \mathrm{d} \xi \mathrm{d} r=\int_{\mathbb{R}^{d} \times \mathbb{R}^{+}} \psi\left(x-x^{\prime}, \xi, r\right)^{2} r^{-h\left(x_{0}\right)} \mathrm{d} \xi \mathrm{d} r .
$$

This allows us to write $\Gamma^{H}$ as $\Gamma^{H}\left(x, x^{\prime}\right)=\frac{1}{2}\left(v(x)+v\left(x^{\prime}\right)-v\left(x-x^{\prime}\right)\right)$, where $v(x)=$ $\Gamma^{H}(x, x)=c\|x\|^{2 H}$ according to Lemma A.2.

This proves the covariance part of the first point of Theorem 3.1. Now let us prove the convergence in finite-dimensional distributions. Let us denote by $\tilde{X}=X-\mathrm{E}(X)$ the centered version of $X$. For the sake of notational simplicity, we will only consider the limit in distribution 
of $\lambda^{-H} \Delta_{x_{0}} \tilde{X}(\lambda x)$ for a fixed $x$ in $\mathbb{R}^{d}$, instead of a random vector $\left(\lambda^{-H} \Delta_{x_{0}} \tilde{X}\left(\lambda x_{j}\right)\right)_{1 \leq j \leq n}$. The general case follows along the same lines. For $H>0, x \in \mathbb{R}^{d}$, and $s \in \mathbb{R}$, let us write

$$
\operatorname{Eexp}\left[\text { is } \frac{\Delta_{x_{0}} \tilde{X}(\lambda x)}{\lambda^{H}}\right]=\exp \left[\Phi\left(H, \lambda, x_{0}, x, s\right)\right]
$$

where $\Phi\left(H, \lambda, x_{0}, x, s\right)$ is given by

$$
\int_{\mathbb{R}^{d} \times \mathbb{R}^{+}}\left(\exp \left[\mathrm{i} s \lambda^{-H} \psi\left(\lambda x, \xi-x_{0}, r\right)\right]-1-\mathrm{i} s \lambda^{-H} \psi\left(\lambda x, \xi-x_{0}, r\right)\right) F(\xi, r) \mathrm{d} \xi \mathrm{d} r .
$$

By a change of variables, $\Phi\left(H, \lambda, x_{0}, x, s\right)$ is equal to

$$
\int_{\mathbb{R}^{d} \times \mathbb{R}^{+}} \lambda^{d+1}\left(\exp \left[\mathrm{i} s \lambda^{-H} \psi(x, \xi, r)\right]-1-\mathrm{i} s \lambda^{-H} \psi(x, \xi, r)\right) F\left(x_{0}+\lambda \xi, \lambda r\right) \mathrm{d} \xi \mathrm{d} r .
$$

Lemma A.2 allows us to split the integral into $\Phi=\Phi_{1}+\left(\Phi-\Phi_{1}\right)$, where $\Phi_{1}\left(H, \lambda, x_{0}, x, s\right)$ is equal to

$$
\int_{\mathbb{R}^{d} \times \mathbb{R}^{+}} \lambda^{d+1}\left(\exp \left[\mathrm{is} \lambda^{-H} \psi(x, \xi, r)\right]-1\right) F\left(x_{0}+\lambda \xi, \lambda r\right) \mathrm{d} \xi \mathrm{d} r .
$$

Let us set $f_{\lambda}(\xi, r)=\exp \left[\operatorname{is} \lambda^{-H} \psi(x, \xi, r)\right]-1$. Using (3.2), we note that

$$
\left|f_{\lambda}(\xi, r)\right| \leq 2 \times \mathbf{1}_{\{\psi(x, \xi, r) \neq 0\}}=2|\psi(x, \xi, r)| .
$$

Then $\lambda^{d+1} f_{\lambda}(\xi, r) F\left(x_{0}+\lambda \xi, \lambda r\right) \rightarrow 0$ as $\lambda \downarrow 0$, since $F(\xi, r) \leq C r^{-M}$ with $M<d+1$. Corollary A.1 implies that

$$
\lim _{\lambda \downarrow 0} \Phi_{1}\left(H, \lambda, x_{0}, x, t\right)=0 .
$$

The second term, $\Phi_{2}=\Phi-\Phi_{1}$, is given by

$$
-\mathrm{i} s \int_{\mathbb{R}^{d} \times \mathbb{R}^{+}} \lambda^{d+1-H} \psi(x, \xi, r) F\left(x_{0}+\lambda \xi, \lambda r\right) \mathrm{d} \xi \mathrm{d} r .
$$

Corollary A.1 with $f_{\lambda}(\xi, r)=\psi(x, \xi, r), F_{0}(\xi, r)=r^{-h\left(x_{0}\right)}$, and $H=d+1-h\left(x_{0}\right)$ implies that

$$
\lim _{\lambda \downarrow 0} \Phi_{2}\left(H, \lambda, x_{0}, x, s\right)=-\mathrm{i} s \int_{\mathbb{R}^{d} \times \mathbb{R}^{+}} \psi(x, \xi, r) r^{-h\left(x_{0}\right)} \mathrm{d} \xi \mathrm{d} r=0,
$$

since

$$
\lambda^{d+1-H} f_{\lambda}(\xi, r) F_{0}(\lambda \xi, \lambda r) \rightarrow \psi(x, \xi, r) r^{-h\left(x_{0}\right)} \quad \text { as } \lambda \downarrow 0 .
$$

This proves that $H_{\mathrm{FDD}}\left(X, x_{0}\right) \geq H=2 H_{\mathrm{COV}}\left(X, x_{0}\right)$.

Now let us prove the LASS properties of the windowed X-ray transform. As for the model itself, we begin with the COV-LASS property. Let $d \geq 2$ and $\alpha \in S^{d-1}$. For $y_{0}, y \in\langle\alpha\rangle^{\perp}$, let us consider

$$
\Delta_{y_{0}} \mathcal{P}_{\alpha} X(y)=\mathcal{P}_{\alpha} X\left(y_{0}+y\right)-\mathcal{P}_{\alpha} X\left(y_{0}\right)=\int_{\mathbb{R}^{d} \times \mathbb{R}^{+}} G_{\rho}\left(y, \xi-y_{0}, r\right) N(\mathrm{~d} \xi, \mathrm{d} r),
$$

where

$$
G_{\rho}(y, \xi, r)=\int_{\mathbb{R}} \psi(y, \xi-p \alpha, r) \rho(p) \mathrm{d} p
$$


with $\psi$ as given by (3.2). Let us write $\xi=\gamma+t \alpha$, with $\gamma \in\langle\alpha\rangle^{\perp}$ and $t \in \mathbb{R}$, and denote by $\rho_{t}$ the window obtained by translation of $t$, namely $\rho_{t}(p)=\rho(t+p)$. By a change of variable, $G_{\rho}(y, \xi, r)=G_{\rho_{t}}(y, \gamma, r)$. Let us denote by $K_{\lambda}^{H}\left(y_{0}, \cdot\right)$ the covariance function of $\lambda^{-H} \Delta_{y_{0}} \mathcal{P}_{\alpha} X(\lambda \cdot)$ for $H \in(0,1)$ and $y_{0} \in\langle\alpha\rangle^{\perp}$. Then $K_{\lambda}^{H}\left(y_{0}, y, y^{\prime}\right)=\int_{\langle\alpha\rangle^{\perp} \times \mathbb{R} \times \mathbb{R}^{+}} \lambda^{-2 H} G_{\rho_{t}}\left(\lambda y, \gamma-y_{0}, r\right) G_{\rho_{t}}\left(\lambda y^{\prime}, \gamma-y_{0}, r\right) F(\gamma+t \alpha, r) \mathrm{d} \gamma \mathrm{d} t \mathrm{~d} r$ for $y, y^{\prime} \in\langle\alpha\rangle^{\perp}$. Let us remark that $G_{\rho_{t}}(\lambda y, \lambda \gamma, \lambda r)=\lambda G_{\rho_{t}(\lambda .)}(y, \gamma, r)$ by a change of variables. Thus, $K_{\lambda}^{H}\left(y_{0}, y, y^{\prime}\right)$ is equal to

$$
\int_{\langle\alpha\rangle \perp \times \mathbb{R} \times \mathbb{R}^{+}} \lambda^{-2 H+d+2} G_{\rho_{t}(\lambda \cdot)}(y, \gamma, r) G_{\rho_{t}(\lambda \cdot)}\left(y^{\prime}, \gamma, r\right) F\left(y_{0}+t \alpha+\lambda \gamma, \lambda r\right) \mathrm{d} \gamma \mathrm{d} t \mathrm{~d} r,
$$

which can be written as

$$
\begin{aligned}
\int_{\mathbb{R} \times\langle\alpha\rangle^{\perp} \times \mathbb{R} \times \mathbb{R}^{+}} & \lambda^{-2 H+d+2} \psi(y, \gamma-p \alpha, r) \rho_{t}(\lambda p) \\
& \times G_{\rho_{t}(\lambda \cdot)}\left(y^{\prime}, \gamma, r\right) F\left(y_{0}+t \alpha+\lambda \gamma, \lambda r\right) \mathrm{d} p \mathrm{~d} \gamma \mathrm{d} t \mathrm{~d} r .
\end{aligned}
$$

Let us set $t^{\prime}=t+\lambda p$ and $\xi=\gamma-p \alpha \in \mathbb{R}^{d} \cong\langle\alpha\rangle^{\perp} \times \mathbb{R}$. In this notation, we can write $K_{\lambda}^{H}\left(y_{0}, y, y^{\prime}\right)$ as

$$
\int_{\mathbb{R}^{d} \times \mathbb{R}^{+} \times \mathbb{R}} \lambda^{-2 H+d+2} \psi(y, \xi, r) G_{\rho_{t^{\prime}}(\lambda \cdot)}\left(y^{\prime}, \xi, r\right) F\left(y_{0}+t^{\prime} \alpha+\lambda \xi, \lambda r\right) \rho\left(t^{\prime}\right) \mathrm{d} \xi \mathrm{d} r \mathrm{~d} t^{\prime} .
$$

In order to apply Lemma A.1 and Corollary A.1, let us check assumptions (A.2) and (A.3) for

$$
f_{\lambda}(\xi, r)=\psi(y, \xi, r) G_{\rho_{t^{\prime}}(\lambda \cdot)}\left(y^{\prime}, \xi, r\right) .
$$

We remark that, in the special case where $\rho \equiv 1$, writing $G$ instead of $G_{1}$, a simple computation gives

$$
G(y, \gamma, r) \equiv G_{1}(y, \gamma, r)=\left(r^{2}-\|y-\gamma\|^{2}\right)_{+}^{1 / 2}-\left(r^{2}-\|\gamma\|^{2}\right)_{+}^{1 / 2}
$$

for $y, \gamma$ in $\langle\alpha\rangle^{\perp}$ and $r$ in $\mathbb{R}^{+}$, where, as usual, $t_{+}:=\max (0, t)$ for all $t \in \mathbb{R}$.

Lemma A.4 provides upper bounds for the integral of $G(y, \cdot)$. We first provide a preliminary result necessary in its proof.

Lemma A.3. Let $n \in \mathbb{N}$ with $n \geq 1$. There exists a constant $C>0$ such that

$$
\int_{\mathbb{R}^{n}}\left|\left(r^{2}-\|x-e\|^{2}\right)_{+}^{1 / 2}-\left(r^{2}-\|x+e\|^{2}\right)_{+}^{1 / 2}\right|^{2} \mathrm{~d} x \leq C r^{n} \ln (2+r),
$$

for all directions $e \in S^{n-1}$ and all $r>0$.

Proof. For $n=1$, we have to prove that there exists a constant $C$ such that, for $r>0$,

$$
\int_{0}^{r+1}\left|\left(r^{2}-(x-1)^{2}\right)_{+}^{1 / 2}-\left(r^{2}-(x+1)^{2}\right)_{+}^{1 / 2}\right|^{2} \mathrm{~d} x \leq C r \ln (r+2) .
$$

This is an easy consequence of the fact that the function that we integrate is bounded by $4 r$ for $x \in[r-1, r+1]$ and by $16 r^{2}((r-1)(r-x+1))^{-1}$ for $x \in[0, r-1]$ when $r>1$. 
In the general case $(n>1)$, we write $x=x^{\prime}+x^{\prime \prime} e$ with $x^{\prime} \in\langle e\rangle^{\perp}$ and $x^{\prime \prime} \in \mathbb{R}$. From the one-dimensional case, for $x^{\prime} \in\langle e\rangle^{\perp}$,

$$
\begin{aligned}
\int_{\mathbb{R}}\left|\left(r^{2}-\left\|x^{\prime}\right\|^{2}-\left|x^{\prime \prime}-1\right|^{2}\right)_{+}^{1 / 2}-\left(r^{2}-\left\|x^{\prime}\right\|^{2}-\left|x^{\prime \prime}+1\right|^{2}\right)_{+}^{1 / 2}\right|^{2} \mathrm{~d} x^{\prime \prime} & \\
& \leq C\left(r^{2}-\left\|x^{\prime}\right\|^{2}\right)_{+}^{1 / 2} \ln (r+2) .
\end{aligned}
$$

However,

$$
\int_{\langle e\rangle^{\perp}}\left(r^{2}-\left\|x^{\prime}\right\|^{2}\right)_{+}^{1 / 2} \mathrm{~d} x^{\prime}=r^{n}\left|S^{n-2}\right| \int_{0}^{1}\left(1-t^{2}\right)^{1 / 2} t^{n-2} \mathrm{~d} t .
$$

Finally, we can change the constant $C$ such that

$$
\int_{\mathbb{R}^{n}}\left|\left(r^{2}-\|x-e\|^{2}\right)_{+}^{1 / 2}-\left(r^{2}-\|x+e\|^{2}\right)_{+}^{1 / 2}\right|^{2} \mathrm{~d} x \leq C r^{n} \ln (2+r),
$$

which concludes the proof.

Lemma A.4. Let $M \in(d, d+2)$. There exists a constant $C_{M} \in(0, \infty)$ such that, for all $y \in\langle\alpha\rangle^{\perp}$,

$$
\int_{\langle\alpha\rangle \perp \times \mathbb{R}^{+}} G(y, \gamma, r)^{2} r^{-M} \mathrm{~d} \gamma \mathrm{d} r=C_{M}\|y\|^{d+2-M}
$$

with

$$
\int_{\langle\alpha\rangle} G(y, \gamma, r)^{2} \mathrm{~d} \gamma \leq C(y) \min \left(r^{d+1}, r^{d-1}|\ln (r)|\right) .
$$

Proof. For $y \in\langle\alpha\rangle^{\perp}$, on the one hand,

$$
\int_{\langle\alpha\rangle^{\perp}} G(y, \gamma, r)^{2} \mathrm{~d} \gamma \leq r^{2} \int_{\langle\alpha\rangle^{\perp}}\left(\mathbf{1}_{\{\|y-\gamma\|<r\}}+\mathbf{1}_{\{\|\gamma\|<r\}}\right) \mathrm{d} \gamma \leq C r^{d+1} .
$$

On the other hand, for $y \neq 0$ and $r>0$, a change of variables gives

$$
\begin{aligned}
& \left(\frac{\|y\|}{2}\right)^{-(d+1)} \int_{\langle\alpha\rangle^{\perp}} G(y, \gamma, r)^{2} \mathrm{~d} \gamma \\
& \quad=\int_{\langle\alpha\rangle^{\perp}}\left|\left(\left(\frac{2 r}{\|y\|}\right)^{2}-\left\|\gamma-\frac{y}{\|y\|}\right\|_{+}^{2}\right)_{+}^{1 / 2}-\left(\left(\frac{2 r}{\|y\|}\right)^{2}-\left\|\gamma+\frac{y}{\|y\|}\right\|^{2}\right)_{+}^{1 / 2}\right|^{2} \mathrm{~d} \gamma .
\end{aligned}
$$

Lemma A.3 provides an upper bound for the last quantity: we obtain

$$
\int_{\langle\alpha\rangle^{\perp}} G(y, \gamma, r)^{2} \mathrm{~d} \gamma \leq C\|y\|^{2} r^{d-1} \ln \left(2+\frac{2 r}{\|y\|}\right) .
$$

Since $M \in(d, d+1)$, inequalities (A.12) and (A.13) imply the integrability of $G^{2}$. The result, (A.10), is then obtained by homogeneity as in the proof of Lemma A.2.

Let us verify that $f_{\lambda}$, given by (A.9), satisfies the assumptions of Lemma A.1. First let us remark that $\left|G_{\rho_{t^{\prime}}(\lambda \cdot)}\left(y^{\prime}, \xi, r\right)\right| \leq\|\rho\|_{\infty}\left|G\left(y^{\prime}, \gamma, r\right)\right|$, meaning that $f_{\lambda}$ is bounded by $f_{\infty}(\xi, r)=$ $\|\rho\|_{\infty}\left|\psi(y, \xi, r) G\left(y^{\prime}, \xi, r\right)\right|$, with $\|\rho\|_{\infty}=\sup _{t \in \mathbb{R}}|\rho|$. Moreover,

$$
\int_{\mathbb{R}}\left|f_{\infty}(\gamma+p \alpha, r)\right| \mathrm{d} p \leq\|\rho\|_{\infty}|G(y, \gamma, r)| \times\left|G\left(y^{\prime}, \gamma, r\right)\right|,
$$


and (A.11) implies (A.2) for all $q_{0}>d$ and $q_{1}=d+2$. In order to prove (A.3), let us note that, for $A>0$ large enough in comparison with $y, \psi(y, \xi, r)=0$ for $\|\xi\|>A$ and $r \leq A / 2$. Hence,

$$
\begin{aligned}
\int_{\|\xi\|>A} \int_{\mathbb{R}^{+}}\left|f_{\infty}(\xi, r)\right| r^{-M} \mathrm{~d} \xi \mathrm{d} r & \leq \int_{A / 2}^{\infty} \int_{\mathbb{R}^{d}}\left|f_{\infty}(\xi, r)\right| r^{-M} \mathrm{~d} r \mathrm{~d} \xi \\
& \leq C\left(y, y^{\prime}\right) A^{q_{0}-M}
\end{aligned}
$$

for any $q_{0} \in(d, M)$, according to (A.2). Then Lemma A.1 holds with $F_{0}(\xi, r)=r^{-h\left(y_{0}+t^{\prime} \alpha\right)}$. Moreover, for $M\left(\alpha, y_{0}\right)=\sup \left\{h\left(y_{0}+t \alpha\right): t \in \operatorname{supp} \rho\right\}$, as $\lambda$ decreases to 0 ,

$$
\begin{aligned}
\lambda^{M\left(\alpha, y_{0}\right)} f_{\lambda}(\xi, r) F_{0}(\lambda \xi, \lambda r) \rho\left(t^{\prime}\right) & \\
& \rightarrow \psi(y, \xi, r) G\left(y^{\prime}, \xi, r\right) r^{-M\left(\alpha, y_{0}\right)} \rho\left(t^{\prime}\right)^{2} \mathbf{1}_{\left\{h\left(y_{0}+t^{\prime} \alpha\right)=M\left(\alpha, y_{0}\right)\right\}} .
\end{aligned}
$$

By Corollary A.1 and then Lebesgue's theorem, for $-2 H+d+2=M\left(\alpha, y_{0}\right)$,

$$
K_{\lambda}^{H}\left(y_{0}, y, y^{\prime}\right) \rightarrow\left(\int_{\mathbb{R}} \mathbf{1}_{\left\{h\left(y_{0}+t^{\prime} \alpha\right)=M\left(\alpha, y_{0}\right)\right\}} \rho^{2}\left(t^{\prime}\right) \mathrm{d} t^{\prime}\right) K^{H}\left(y, y^{\prime}\right) \quad \text { as } \lambda \downarrow 0,
$$

where

$$
K^{H}\left(y, y^{\prime}\right)=\int_{\langle\alpha\rangle^{\perp} \times \mathbb{R}^{+}} G(y, \gamma, r) G\left(y^{\prime}, \gamma, r\right) r^{d+2-2 H} \mathrm{~d} \gamma \mathrm{d} r .
$$

The identification of $K^{H}$ as the covariance of a fractional Brownian motion defined on $\langle\alpha\rangle^{\perp}$ with Hurst index $H=\left(d+2-M\left(\alpha, y_{0}\right)\right) / 2$ is straightforward following the same arguments as for the random-balls model itself.

If $\left\{t \in \operatorname{supp} \rho: h\left(y_{0}+t \alpha\right)=M\left(\alpha, y_{0}\right)\right\}$ has positive measure then the proof of the COVLASS property is complete. Otherwise, it is sufficient to remark that, following the same lines as in the proofs of Lemma A.1 and Corollary A.1, for all $\varepsilon>0$ and $H=\left(d+2-M\left(\alpha, y_{0}\right)\right) / 2+\varepsilon$ we can find a $C>0$ such that, for small enough $\lambda$, a lower bound of $K_{\lambda}^{H}\left(y_{0}, y, y\right)$ is given by

$$
C \int_{\mathbb{R}^{d} \times \mathbb{R}^{+} \times \mathbb{R}} \lambda^{-\varepsilon} G(y, \gamma, r)^{2} r^{-M\left(\alpha, y_{0}\right)+\varepsilon} \mathbf{1}_{\left\{h\left(y_{0}+t^{\prime} \alpha\right) \geq M\left(\alpha, y_{0}\right)-\varepsilon\right\}} \rho\left(t^{\prime}\right)^{2} \mathrm{~d} \xi \mathrm{d} r \mathrm{~d} t^{\prime} .
$$

This proves that $H_{\mathrm{COV}}\left(\mathcal{P}_{\alpha} X, y_{0}\right) \leq H$ and yields the result.

Finally, we consider the FDD-LASS property at point $y_{0}$ for the X-ray transform. As above, we restrict the computation to the one-dimensional distribution and denote by $\overline{\mathcal{P}_{\alpha} X}=$ $\mathcal{P}_{\alpha} X-\mathrm{E}\left(\mathcal{P}_{\alpha} X\right)$ the centered version of $\mathcal{P}_{\alpha} X$. For any $y \in\langle\alpha\rangle^{\perp}, s \in \mathbb{R}$, and $H \in(0,1)$, we write

$$
\operatorname{Eexp}\left[i s \lambda^{-H}\left(\widetilde{\mathcal{P}_{\alpha} X}\left(y_{0}+\lambda y\right)-\widetilde{\mathcal{P}_{\alpha} X}\left(y_{0}\right)\right)\right]=\exp \left[\Phi\left(H, \lambda, y_{0}, y, s\right)\right],
$$

where $\Phi\left(H, \lambda, y_{0}, y, s\right)$ is given by

$$
\int_{\mathbb{R}^{d} \times \mathbb{R}^{+}}\left(\exp \left[\mathrm{i} s \lambda^{-H} G_{\rho}\left(\lambda y, \xi-y_{0}, r\right)\right]-1-\mathrm{i} s \lambda^{-H} G_{\rho}\left(\lambda y, \xi-y_{0}, r\right)\right) F(\xi, r) \mathrm{d} \xi \mathrm{d} r .
$$

By the same change of variables as in the covariance part of the proof, $\Phi\left(H, \lambda, y_{0}, y, s\right)$ is equal to

$$
\begin{aligned}
\int_{\langle\alpha\rangle \perp \times \mathbb{R} \times \mathbb{R}^{+}} & \lambda^{d}\left(\exp \left[i s \lambda^{1-H} G_{\rho_{t}(\lambda \cdot)}(y, \gamma, r)\right]-1-i s \lambda^{1-H} G_{\rho_{t}(\lambda \cdot)}(y, \gamma, r)\right) \\
& \times F\left(\lambda \gamma+t \alpha+y_{0}, \lambda r\right) \mathrm{d} \gamma \mathrm{d} t \mathrm{~d} r .
\end{aligned}
$$


Let us remark that, for $\delta \in[0,1]$,

$$
\begin{array}{r}
\left|\exp \left[\mathrm{i} s \lambda^{1-H} G_{\rho_{t}(\lambda \cdot)}(y, \gamma, r)\right]-1-\mathrm{i} s \lambda^{1-H} G_{\rho_{t}(\lambda \cdot)}(y, \gamma, r)+\frac{s^{2}}{2} \lambda^{2-2 H} G_{\rho_{t}(\lambda \cdot)}(y, \gamma, r)^{2}\right| \\
\leq C(s) \lambda^{(1-H)(2+\delta)} G_{\rho_{t}(\lambda \cdot)}(y, \gamma, r)^{2+\delta} .
\end{array}
$$

Following the same kind of computations as in the proof of the COV-LASS property, we replace $f_{\lambda}$, from (A.9), by $f_{\lambda}(\xi, r)=\psi(y, \xi, r) G_{\rho_{t^{\prime}}(\lambda \cdot)}^{1+\delta}(y, \xi, r)$. Since $\left|G_{\rho_{t}(\lambda \cdot)}(y, \gamma, r)\right| \leq C r$, by choosing $\delta \in(0, M-d)$ for $H=\left(d+2-M\left(\alpha, y_{0}\right)\right) / 2$ we obtain

$$
\lim _{\lambda \downarrow 0} \int_{\langle\alpha\rangle^{\perp} \times \mathbb{R} \times \mathbb{R}^{+}} \lambda^{d+2-2 H+\delta(1-H)} G_{\rho_{t}(\lambda \cdot)}(y, \gamma, r)^{2+\delta} F\left(\lambda \gamma+t \alpha+y_{0}, \lambda r\right) \mathrm{d} \gamma \mathrm{d} t \mathrm{~d} r=0 .
$$

Therefore, as $\lambda$ decreases to 0 ,

$$
\Phi\left(H, \lambda, y_{0}, y, s\right) \rightarrow-\frac{1}{2} s^{2}\left(\int_{\mathbb{R}} \mathbf{1}_{\left\{h\left(y_{0}+t^{\prime} \alpha\right)=M\left(\alpha, y_{0}\right)\right\}} \rho\left(t^{\prime}\right)^{2} \mathrm{~d} t^{\prime}\right) \Gamma^{H}(y, y),
$$

which concludes the proof.

\section{A.2. Proof of Theorem 3.2}

We now assume that $h$ is given by an even $\beta$-Hölder function on the sphere, extended onto $\mathbb{R}^{d} \backslash\{0\}$ by taking $h(\xi)=h(\xi /\|\xi\|)$.

Let us prove the COV-LASS property for the random-balls model at point $x_{0}=0$. We retain the notation of Section A.1. We have

$$
\Gamma_{\lambda}^{H}\left(0, x, x^{\prime}\right)=\int_{\mathbb{R}^{d} \times \mathbb{R}^{+}} \lambda^{-2 H+d+1} \psi(\lambda x, \lambda \xi, \lambda r) \psi\left(\lambda x^{\prime}, \lambda \xi, \lambda r\right) F(\lambda \xi, \lambda r) \mathrm{d} \xi \mathrm{d} r .
$$

In this case, $F$ satisfies the assumption of Lemma A.1 with $F_{0}(\xi, r)=r^{-h(\xi /\|\xi\|)}$. For $M=$ $\max _{S^{d-1}} h,(\mathrm{~A} .7)$ is replaced by

$$
\lambda^{M} f_{\lambda}(\xi, r) F_{0}(\lambda \xi, \lambda r) \rightarrow f_{0}(\xi, r) r^{-M} \mathbf{1}_{\{h(\xi)=M\}} \quad \text { as } \lambda \downarrow 0 .
$$

Thus, using Corollary A.1, we find that $\Gamma_{\lambda}^{H}\left(0, x, x^{\prime}\right)$ tends to

$$
\int_{\mathbb{R}^{d} \times \mathbb{R}^{+}} \mathbf{1}_{\{h(\xi)=M\}} \psi(x, \xi, r) \psi\left(x^{\prime}, \xi, r\right) r^{-d-1+2 H} \mathrm{~d} \xi \mathrm{d} r=: \Gamma_{H}\left(x, x^{\prime}\right)
$$

for $-2 H+d+1=M$. Let us remark that $\Gamma_{H}$ vanishes if and only if $\left\{\xi \in \mathbb{R}^{d} \backslash\{0\}: h(\xi)=M\right\}$ has measure 0 . In this case, for $d+1-2 H=M-2 \varepsilon$ with $\varepsilon \in(0,(M-q) / 2)$ and for $\lambda$ small enough, Lemma A.1 yields

$$
\Gamma_{\lambda}^{H}(0, x, x) \geq \frac{\lambda^{-\varepsilon}}{2} \int_{\mathbb{R}^{d} \times(0,1)} \mathbf{1}_{\{h(\xi)>M-\varepsilon\}} \psi(x, \xi, r)^{2} r^{-h(\xi)} \mathrm{d} \xi \mathrm{d} r-C \lambda^{M-2 \varepsilon-q},
$$

where $\left\{\xi \in \mathbb{R}^{d} \backslash\{0\}: h(\xi)>M-\varepsilon\right\}$ has positive measure. The above quantity thus tends to $\infty$ as $\lambda$ decreases to 0 . Hence, the exponent $H=(d+1-M) / 2$ is proved to be the COV-LASS index for $X$ at $x_{0}=0$. 
Let us now prove the remainder of point one of Theorem 3.2. In the notation of Section A.1, for $H=d+1-M=2 H_{\mathrm{COV}}(X, 0),(\mathrm{A} .8)$ is replaced by

$$
\lambda^{d+1-H} f_{\lambda}(\xi, r) F_{0}(\lambda \xi, \lambda r) \rightarrow \psi(x, \xi, r) r^{-M} \mathbf{1}_{\{h(\xi)=M\}} \quad \text { as } \lambda \downarrow 0,
$$

since $F_{0}(\xi, r)=r^{-h(\xi /\|\xi\|)}$ in this case. By Corollary A.1, we obtain

$$
\begin{aligned}
\lim _{\lambda \downarrow 0} \Phi(H, \lambda, 0, x, s) & =\lim _{\lambda \downarrow 0} \Phi_{2}(H, \lambda, 0, x, s) \\
& =-\mathrm{i} s \int_{\mathbb{R}^{d} \times \mathbb{R}^{+}} \mathbf{1}_{\{h(\xi)=M\}} \psi(x, \xi, r) r^{-d-1+H} \mathrm{~d} \xi \mathrm{d} r .
\end{aligned}
$$

In the following, we will distinguish between two cases according to whether or not $\{\xi: h(\xi)=$ $M\}$ has positive measure.

First assume that $\{\xi: h(\xi)=M\}$ has positive measure. Hence, the finite-dimensional distributions of $\lambda^{-H} \Delta_{0} X(\lambda \cdot)$ converge to the finite-dimensional distributions of the deterministic field

$$
Z_{H}(x)=-\int_{\mathbb{R}^{d} \times \mathbb{R}^{+}} \mathbf{1}_{\{h(\xi)=M\}} \psi(x, \xi, r) r^{-d-1+H} \mathrm{~d} \xi \mathrm{d} r, \quad x \in \mathbb{R}^{d} .
$$

It remains to show that $Z_{H}$ is nonzero. This follows from the next lemma since, by continuity of $h,\{\xi: h(\xi) \neq M\}$ contains a ball.

Lemma A.5. Let $M \in(d, d+1)$. For all Borel sets $E \subset \mathbb{R}^{d}$ with positive measure, if

$$
\int_{\mathbb{R}^{d} \times \mathbb{R}^{+}} \mathbf{1}_{E}(\xi) \psi(x, \xi, r) r^{-M} \mathrm{~d} \xi \mathrm{d} r=0
$$

for all $x \in \mathbb{R}^{d}$, then the (complement) set $E^{\mathrm{c}}$ does not contain an open ball.

Proof. Let us write $I_{E}^{M}(x)=\int_{\mathbb{R}^{d} \times \mathbb{R}^{+}} \mathbf{1}_{E}(\xi) \psi(x, \xi, r) r^{-M} \mathrm{~d} \xi \mathrm{d} r$ for $x \in \mathbb{R}^{d}$. Note that

$$
\int_{\mathbb{R}^{+}} \psi(x, \xi, r) r^{-M} \mathrm{~d} \xi \mathrm{d} r=(M-1)^{-1}\left(\|x-\xi\|^{-M+1}-\|\xi\|^{-M+1}\right) .
$$

Then, for all Borel sets $E \subset \mathbb{R}^{d}$,

$$
I_{E}^{M}(x)=\int_{\mathbb{R}^{d}} \mathbf{1}_{E}(\xi)(M-1)^{-1}\left(\|x-\xi\|^{-M+1}-\|\xi\|^{-M+1}\right) \mathrm{d} \xi .
$$

Let us suppose that we can find an open, nonempty ball $B \subset E^{\mathrm{c}}$. Then $I_{E}^{M}$ is smooth on $B$. The Laplacian of $I_{E}^{M}$ is

$$
\Delta I_{E}^{M}(x)=(M+1-d) \int_{\mathbb{R}^{d}} \mathbf{1}_{E}(\xi)\|x-\xi\|^{-M-1} \mathrm{~d} \xi
$$

and proves to be positive on $B$. Thus, $I_{E}^{M}$ does not vanish on $B$. This completes the proof of Lemma A.5 and, thus, that of Theorem 3.2 in the case where $\{\xi: h(\xi)=M\}$ has positive measure.

Now assume that $\{\xi: h(\xi)=M\}$ is of measure 0 . We will establish that the FDD-LASS index of $X$ at 0 is still equal to $d+1-M$. For $H \leq d+1-M$, we find that $\lim _{\lambda \downarrow 0} \Phi(H, \lambda, 0, x, s)=0$. On the other hand, for $H>d+1-M$, we have to prove that there exists at least one $x \in \mathbb{R}^{d}$ 
such that $\Phi_{2}(H, \lambda, 0, x, s)$ does not tend to 0 . First, let us remark that, from Lemma A.1 and for all $x \in \mathbb{R}^{d}, \Phi_{2}(H, \lambda, 0, x, s)$ is equivalent to $-\mathrm{is} \tilde{\Phi}_{2}(H, \lambda, x)$ as $\lambda$ decreases to 0 , with

$$
\tilde{\Phi}_{2}(H, \lambda, x)=\int_{\mathbb{R}^{d} \times \mathbb{R}^{+}} \lambda^{d+1-H-h(\xi)} \psi(x, \xi, r) r^{-h(\xi)} \mathrm{d} \xi \mathrm{d} r .
$$

However, $\tilde{\Phi}_{2}(H, \lambda, x)$ may be written as $\int_{\mathbb{R}^{d}}(h(\xi)-1)^{-1} \lambda^{d+1-H-h(\xi)} f(x, \xi) \mathrm{d} \xi$ with

$$
f(x, \xi)=\|x-\xi\|^{-h(\xi)+1}-\|\xi\|^{-h(\xi)+1} .
$$

The function $f(\cdot, \xi)$ is smooth on $\mathbb{R}^{d} \backslash\{\xi\}$, and its Laplacian, given by

$$
\Delta f(x, \xi)=(h(\xi)+1-d)(h(\xi)-1)\|x-\xi\|^{-h(\xi)-1},
$$

is positive. Approximating the Laplacian by the second-order increments, we can thus find a $C>0$ such that

$$
\left|\sum_{1 \leq j \leq d} f\left(x+\delta e_{j}, \xi\right)+f\left(x-\delta e_{j}, \xi\right)-2 f(x, \xi)-\frac{\delta^{2}}{2} \Delta f(x, \xi)\right| \leq C \delta^{3}\|x-\xi\|^{-h(\xi)-2}
$$

whenever $\|x-\xi\| \neq 0$ and $\delta \leq \frac{1}{2}\|x-\xi\|$. Let us choose $H$ such that $\min _{S^{d-1}} h<d+$ $1-H<M$, and note that the sets $\{\xi: h(\xi)<d+1-H\}$ and $\{\xi: h(\xi) \geq d+1-H\}$ have positive measures. Moreover, by continuity of $h$, there exists an open, nonempty ball $B \subset\{\xi: h(\xi)<d+1-H\}$. For every $x \in \mathbb{R}^{d}$, we introduce

$$
\tilde{\Phi}_{\lambda}(x):=\int_{\mathbb{R}^{d}} \mathbf{1}_{\{h(\xi) \geq d+1-H\}}(h(\xi)-1)^{-1} \lambda^{d+1-H-h(\xi)} f(x, \xi) \mathrm{d} \xi
$$

and write

$$
\tilde{\Phi}_{2}(H, \lambda, x)=\tilde{\Phi}_{\lambda}(x)+\left(\tilde{\Phi}_{2}(H, \lambda, x)-\tilde{\Phi}_{\lambda}(x)\right) .
$$

The second term tends to 0 with $\lambda$, by Lebesgue's theorem. Suppose that $\tilde{\Phi}_{\lambda}(x)$ tends to 0 with $\lambda$ for every $x$ in $\mathbb{R}^{d}$. Then

$$
\Delta_{\delta}^{(2)} \tilde{\Phi}_{\lambda}(x):=\sum_{1 \leq j \leq d}\left(\tilde{\Phi}_{\lambda}\left(x+\delta e_{j}\right)+\tilde{\Phi}_{\lambda}\left(x-\delta e_{j}\right)-2 \tilde{\Phi}_{\lambda}(x)\right) \rightarrow 0 \quad \text { as } \lambda \downarrow 0,
$$

for all $x \in \mathbb{R}^{d}$ and all $\delta \in \mathbb{R}$. However, for $x \in B$ and a $\delta>0$ small enough that $B(x, \delta) \subset B$, according to (A.14) we have

$$
\Delta_{\delta}^{(2)} \tilde{\Phi}_{\lambda}(x) \geq C(\delta) \int_{\mathbb{R}^{d}} \mathbf{1}_{\{h(\xi) \geq d+1-H\}}(h(\xi)+1-d)\|x-\xi\|^{-h(\xi)-1} \mathrm{~d} \xi \geq 0,
$$

since $\lambda^{d+1-H-h(\xi)} \mathbf{1}_{\{h(\xi) \geq d+1-H\}} \geq \mathbf{1}_{\{h(\xi) \geq d+1-H\}}$. Equation (A.15) then implies that $\{\xi: h(\xi) \geq d+1-H\}$ has measure 0 , which contradicts the assumption that $d+1-H<M$.

The proof of the first part of Theorem 3.2 is now complete.

Let us conclude with the proof of the LASS properties of the windowed X-ray transform. It is sufficient to remark that, for any $t \neq 0$, since $h$ is $\beta$-Hölder on $S^{d-1}$ with $0<\beta \leq 1$, we obtain

$$
|h(t \alpha+x)-h(\alpha)| \leq C|t|^{-\beta}\|x\|^{\beta} .
$$

We can therefore argue in the same way as we did in proving Theorem 3.1, with $y_{0}=0$, $M\left(\alpha, y_{0}\right)=h(\alpha)$, and $\mathbf{1}_{\left\{h\left(y_{0}+t \alpha\right)=M\left(\alpha, y_{0}\right)\right\}}=1$ a.e. 


\section{Acknowledgements}

We would like to thank Aline Bonami warmly for her useful contribution to the simplification of many computations, as well as for very fruitful discussions, and Peter Scheffler for the careful reading of our manuscript. The authors are also very grateful to the referees for improving the first version of this text.

\section{References}

[1] Benassi, A., Cohen, S. And Istas, J. (2003). Local self-similarity and the Hausdorff dimension. C. R. Acad. Sci. Paris Ser. I 336, 267-272.

[2] Benassi, A., Cohen, S. And Istas, J. (2004). On roughness indices for fractional fields. Bernoulli 10, 357-376.

[3] Benassi, A., JafFARD, S. AND Roux, D. (1997). Elliptic Gaussian random processes. Rev. Mat. Iberoamericana 13, 19-89.

[4] Biermé, H. (2005). Champs aléatoires: autosimilarité, anisotropie et étude directionnelle. Doctoral Thesis, University of Orléans, France. Available at http://www.math-info.univ-paris5.fr/ bierme/recherche/Thesehb.pdf.

[5] Biermé, H., Estrade, A. And KaJ, I. (2006). About scaling behavior of random balls models. In Proc. 6th Internat. Conf. Stereology, Spatial Statist. Stoch. Geometry, Prague, Union of Czech Mathematicians and Physicists, pp. 63-68.

[6] Bonami, A. And Estrade, A. (2003). Anisotropic analysis of some Gaussian models. J. Fourier Anal. Appl. 9, 215-236.

[7] Cioczek-Georges, R. and Mandelbrot, B. B. (1995). A class of micropulses and antipersistent fractional Brownian motion. Stoch. Process. Appl. 60, 1-18.

[8] Cohen, S. And TAqQu, M. (2004). Small and large scale behavior of the Poissonized telecom process. Methodology Comput. Appl. Prob. 6, 363-379.

[9] Falconer, K. J. (2003). The local structure of random processes. J. London Math. Soc. 67, 657-672.

[10] HaRba, R. et al. (1994). Determination of fractal scales on trabecular bone X-ray images. Fractals 2, 451-456.

[11] Heinrich, L. ANd SchmidT, V. (1985). Normal convergence of multidimensional shot-noise and rates of this convergence. Adv. Appl. Prob. 17, 709-730.

[12] Kaj, I., Leskelä, L., Norros, I. And Schmidt, V. (2004). Scaling limits for random fields with long-range dependence. To appear in Ann. Prob.

[13] Lacaux, C. (2004). Real harmonizable multifractional Lévy motions. Ann. Inst. H. Poincaré Prob. Statist. 40, 259-277.

[14] Lacaux, C. (2005). Fields with exceptional tangent fields. J. Theoret. Prob. 18, 481-497.

[15] LÉvy-VÉHel, J. ANd Peltier, R. F. (1995). Multifractional Brownian motion: definition and preliminary results. Tech. Rep. 2645, INRIA. Available at http://www.inria.fr/rrt//rr-2645.html.

[16] Ramm, A. G. And Katsevich, A. I. (1996). The Radon Transform and Local Tomography. CRC Press, Boca Raton, FL.

[17] Serra, J. (1982). Image Analysis and Mathematical Morphology. Academic Press, London.

[18] Stoyan, D., Kendall, W. S. And Mecke, J. (1987). Stochastic Geometry and Its Applications. John Wiley, Chichester.

[19] WiCKSELL, S. D. (1925). The corpuscle problem: a mathematical study for a biometrical problem. Biometrika 17, 84-99. 